\title{
A novel effect of geraniin on OPG/RANKL signaling in osteoblasts
}

\author{
Xiaochao Zhang ${ }^{1 \dagger}$, Renhua Yang ${ }^{1 \dagger}$, Jiang Long ${ }^{2 \dagger}$, Bo He ${ }^{1}$, Yue Zhang ${ }^{1}$, Ting Fu ${ }^{1}$, Zhiqiang Shen ${ }^{*}$, \\ Peng Chen ${ }^{*}$
}

\begin{abstract}
${ }^{1}$ School of Pharmaceutical Science \& Yunnan Key Laboratory of Pharmacology for Natural Products, Kunming Medical University, Kunming, P.R. China, ${ }^{2}$ Department of Neurosurgery, the First Affiliated Hospital of Kunming Medical University, Kunming, P.R. China
\end{abstract}

\begin{abstract}
In this study, the effects of geraniin on osteoprotegerin/receptor activator of nuclear factor- $\mathrm{B}$ ligand(OPG/ RANKL) in regulating the proliferation of osteoblasts and suppression of osteoclast-like cells (OLC) in OLC-osteoblast co-cultured system in vitro were investigated. Osteoblasts were cultured and identified with alkaline phosphatase (ALP), gomori stain, and mineralized nodule stain. OLCs were isolated from long bones of Sprague-Dawley (SD) rats and identified with tartrate-resistant acid phosphatase(TRAP) stain. Methyl thiazolyl tetrazolium assay was used to examine the proliferation of osteoblasts, and immunocytochemistry and in situ hybridization to analyze the expression OPG/RANKL in osteoblasts co-cultured with osteoclasts under the action of geraniin, respectively. Geraniin could regulate the proliferation of osteoblasts MC3T3-E1, decrease the number of OLC in OLC-osteoblast co-cultured system, and inhibit the bone resorption areas and resorption pits of OLC in vitro experiments. Geraniin could promote the mRNA and protein expression levels of OPG and suppress those of RANKL in osteoblasts. These results indicate that geraniin has a promoting effect on the proliferation of osteoblasts and an inhibitory effect on the osteoclastic bone-resorption through regulating OPG/RANKL signaling pathway in OLC-OB co-cultured system.
\end{abstract}

Keywords: Geraniin/effect. Osteoblasts. Osteoprotegerin. Receptor activator of nuclear factor $-\kappa$ B ligand.

\section{INTRODUCTION}

Bone morphogenesis and remodeling involve the bone formation from osteoblasts (OB) and the bone resorption by osteoclasts (OC). As an in vitro model of osteoblast differentiation, MC3T3-E1 cell line, derived from newborn murine calvariae and exhibiting a developmental sequence similar to primary osteoblasts, has been examined extensively, and can be identified with hematoxylin and eosin (HE), ALP, Gomori, and mineralized nodules Von Kossa staining, and thus is widely used as to a cytochemical marker that identifies osteoblasts in tissue sections as well as in cell cultures (Ryoo et al., 1997; Lee, Choi, 2011; Kim et al., 2012; Luan et al., 2012; Czekanska et al., 2014). TRAP, a widely used

\footnotetext{
*Correspondences: Z.-Q. Shen, P. Chen. School of Pharmaceutical Science, Yunnan Key Laboratory of Pharmacology for Natural Products, Kunming Medical University, Kunming, 650500, P.R. China. Mobile Number: +861390871 3337. E-mail addresses: shzhq21cn@qq.com (Z.-Q. Shen) / ynkmcp@gmail.com (P. Chen)

$\dagger$ These authors contributed equally to this work
}

cytochemical marker, can identify osteoclasts and their precursors. OLC can be induced from mouse monocyte/ macrophage RAW264.7 that expresses TRAP (Denlinger et al., 1996; Janckila, Yam, 2009).

OPG and RANKL are the important members of tumor necrosis factor (TNF) family found in recent years. OPG is a new open reading frame and encoding a protein with 401 amino acid residues discovered in the analysis of fetal rat intestinal cDNA sequencing by Simonet et al. (1997). OPG is thought to inhibit osteoclastogenesis by disrupting the cell-to-cell signaling between osteoblastic stromal cells and osteoclast progenitors. RANKL is a type II transmembrane protein, and highly expressed in bone and bone marrow. The findings of OPG and RANKL as well as the transgenic characteristics and gene-knockout animals indicate that the cytokine system is the necessary factor for osteoclastic biology (Kiechl et al., 2006).

Geraniin, extracted and separated from the aerial parts of Phyllanthus amarus Linn (Euphorbiaceae), has been found to possess a wide range of biological activities including antioxidation and free radical scavenging (Ito, 
2011), antinociceptive (Kupeli et al., 2007), and antivirus activities (Yang et al., 2012). In the previous experiments, we found that geraniin showed a preventive effect in rat models of tretinoin-induced osteoporosis, and inhibited bone resorption in vitro by down-regulating the mRNA and protein expression of matrix metalloproteinase-9 (MMP-9) in rat osteoclasts (He et al., 2013). However, whether geraniin exhibits an inhibitory effect on bone resorption induced by OLC or modulates OPG/RANKL signal transduction pathway in osteoblasts is not yet known. In this study, we investigated the effect of geraniin on OLCinduced bone resorption in vitro and the mRNA and protein expression of OPG and RANKL in rat osteoblasts.

\section{MATERIAL AND METHODS}

\section{Animals}

All the animal experiments were approved by the Animal Study Committee of Kunming Medical University and were conducted according to the requirements of NIH Guidelines for care and use of laboratory animals. Specific pathogen free (SPF) level, SD rats aged one day, male and female, were purchased from the Animal Center of Kunming Medical University [License: SCXK (Yunnan) 2015-0002].

\section{Drugs and reagents}

Geraniin was extracted and separated from the aerial parts of Phyllanthus amarus Linn (Euphorbiaceae) by Professor Ji-Kai Liu (Kunming Institute of Botany, the Chinese Academy of Sciences, P.R. China). The purity was $99 \%$ and its sample was deposited at Kunming Institute of Botany, the Chinese Academy of Sciences, P.R. China. The chemical construction was shown in Figure1. The compound was dissolved in dimethyl sulfoxide (DMSO) and then diluted with phosphate-buffer saline (PBS) prior to use. The final concentration of geraniin was $0.1 \%$ in PBS, and $0.01 \%$ in DMSO, which is not toxic to osteoclasts or osteoblasts. Alendronate (purity 99\%) was purchased from Langze Medical Science and Technology Company, Nanjing, P.R. China. The compound was dissolved in DMSO and then diluted with PBS prior to use. MC3T3-E1 Subclone 14 was purchased from Cell Bank of the Chinese Academy of Sciences, Shanghai, P.R. China. Mouse monocyte macrophage cell line (RAW264.7) was purchased from Kunming Institute of Zoology, the Chinese Academy of Sciences, Kunming, P.R. China. Bovine bone slices $(10 \times 10 \mathrm{~mm}$ thick) were purchased from West China Medical Center of Sichuan University, Chengdu,
P.R. China. The immunocytochemical kit was purchased from Zhongshan Golden Bridge Biological Company, Beijing, P.R. China. OPG and RANKL mRNA kits were purchased from Boster Bioengineering Company, Wuhan, P.R. China. TRAP-staining kit was purchased from Sigma Chemical Co., St. Louis, MO, USA. HE staining kit and MTT cell proliferation and cytotoxicity detection kit were purchased from Beyotime Biotechnology Research Institute, Jiangsu, P.R. China. The mouse recombinant sRANKL and macrophage colony stimulating factor (M-CSF) were purchased from PeproTech Co., Princeton, NJ, USA. OPG rabbit-anti-mouse polyclonal antibody and RANKL goat-anti-mouse polyclonal antibody were purchased from Santa Cruz Biotechnology, INC. Dallas, TX, USA. Dulbecco's modified eagle medium (DMEM) F-12 and high glucose medium were purchased from HyClone Laboratories Inc. Logan, UT, USA. All the other chemicals were of analytical grade and were purchased from local commercial suppliers.

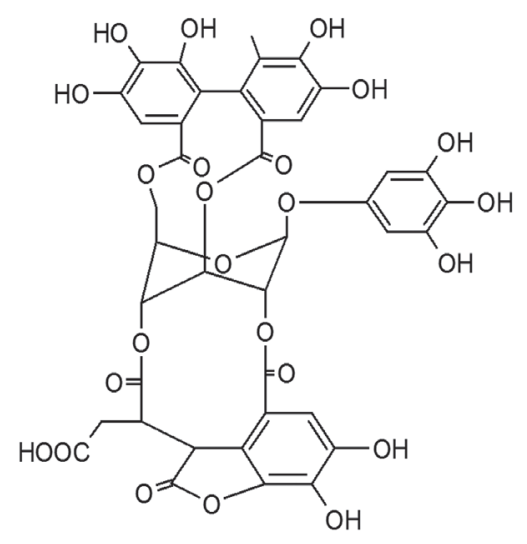

FIGURE 1 - Chemical structure of geraniin.

\section{Culture and identification of osteoblasts}

The detailed culture protocols of osteoblasts were carried out according to the manufacturer's directions. Briefly, after thawing of MC3T3-E1, the cells with $1 \times 10^{6} / \mathrm{mL}$ were inoculated in the culture bottle, with DMEM/F-12 (DMEM: F-12: FBS=9: 9: 2) as the medium, and the cells were cultured under a humidified atmosphere of $5 \% \mathrm{CO}_{2}$ at $37{ }^{\circ} \mathrm{C}$ for $24 \mathrm{~h}$ to allow the osteoblasts to adhere to 24 -well plate directly and began to count, then exchanged the cell culture medium and the remaining adherent cells on the plate were continuously cultured and counted for $7 \mathrm{~d}$, respectively.

The cultured osteoblasts were identified with an invert microscope, HE stain, alkaline phosphorase (ALP) stain, Gomori stain, and stain of mineralized nodule (Von Kossa), respectively. 


\section{Methyl thiazolyl tetrazolium assay}

The proliferation of osteoblasts was examined with methyl thiazolyl tetrazolium (MTT) in microplate reader $(490 \mathrm{~nm})$. Briefly, the cells of the fourth generation were cultured in $1 \times 10^{5} \mathrm{cell} / \mathrm{mL}$ on the 96 -well culture plate with DMEM/F-12 medium for $24 \mathrm{~h}$, and then incubated with the different concentrations of geraniin for $24 \mathrm{~h}$, adding MTT reagent and DMSO, counted the number of cells and measured cell optical density (OD). Osteogenic MC3T3-E1 were divided into nine groups as follows ( $n=6$ per group): blank control group with the complete culture media (later referred to as Control); geraniin groups with $10^{-6}, 10^{-7}, 10^{-8}, 10^{-9}, 10^{-10}, 10^{-11}$, and $10^{-12} \mathrm{~mol} / \mathrm{L}$ of geraniin in osteoblasts (later referred to as Geraniin); positive control group with $10^{-8} \mathrm{~mol} / \mathrm{L}$ of alendronate in osteoblasts (later referred to as Alendronate). The inhibitory rate of cell growth was calculated according to the following formula:

Inhibitory rate $(\%)=[(\mathrm{OD}$ of blank control group (OD of drug-treated group) / OD of blank control group $] \times 100$

\section{Isolation, culture condition and identification of osteoclasts}

Osteoclasts were isolated from the long bones of 1-day-old SD rats. Briefly, SD rats were euthanized via cervical dislocation and their femora were separated. The long bones were dissected free of the adherent soft tissues and quickly minced with a scalpel blade in HEPESbuffered medium 199 supplemented with $15 \%$ fetal calf serum, $100 \mathrm{U} / \mathrm{mL}$ benzyl penicillin, and $100 \mathrm{U} / \mathrm{mL}$ streptomycin. The long bones were homogenized gently, and the cell suspension was collected in a centrifuge tube. The cell suspension was allowed to settle for $10 \mathrm{~s}$, before the supernatant was dropped onto the plastic substrate. This osteoclast-rich supernatant was collected again and filtered through 70- $\mu \mathrm{m}$ nylon filters.

The cells of RAW2 64.7 were cultured under a humidified atmosphere of $5 \% \mathrm{CO}_{2}$ at $37^{\circ} \mathrm{C}$ in DMEM supplemented with $50 \mathrm{U} / \mathrm{mL}$ benzyl penicillin, and 50 $\mathrm{U} / \mathrm{mL}$ streptomycin. After thawing of RAW264.7, the cells were cultured under a humidified atmosphere of 5\% $\mathrm{CO}_{2}$ at $37{ }^{\circ} \mathrm{C}$ in HEPES-buffered DMEM with $15 \%$ fetal calf serum, $100 \mathrm{U} / \mathrm{mL}$ benzyl penicillin, and $100 \mathrm{U} / \mathrm{mL}$ streptomycin.

The detailed protocols of TRAP were carried out according to the manufacturer's directions. Briefly, after cultivation at $37^{\circ} \mathrm{C}$ for $72 \mathrm{~h}$, the cells were fixed with $2.5 \%$ glutaraldehyde solution for $10 \mathrm{~min}$, washed thrice with deionized water, and then incubated in TRAP-staining solution for $50 \mathrm{~min}$. The morphology and number of TRAP-positive cells of OC and OLC were then counted and observed by microscope(Olympus) in $4 \mathrm{~d}, 7 \mathrm{~d}, 12 \mathrm{~d}$, and $15 \mathrm{~d}$, respectively. The TRAP-positive cells possessing three or more nuclei were defined as mature osteoclasts (mOC), and TRAP-positive cells containing less than three nuclei were distinguished as pre-osteoclasts (pOC) ( $n=4$ per group). The fusion index (FI) was calculated as the percentage of the total number of mOC divided by the total number of TRAP-positive cells counted. The inhibitory rate of FI was calculated according to the following formula:

The inhibitory rate of FI $(\%)=[($ FI of blank control group (FI of drug-treated group) / FI of blank control group] $\times 100$

\section{Co-culture of osteoclasts with osteoblasts}

The in vitro establishment of OLC-OB co-cultured system was to inoculate RAW264.7 to lower chambers of 24-well suspension culture plate directly or bovine bone slices for culture together in DMEM medium with 100 $\mathrm{ng} / \mathrm{mL}$ RANKL, $100 \mathrm{ng} / \mathrm{mL}$ M- CSF, and $10^{-8}$ Vitamin $\mathrm{D}_{3}$ $\left(\mathrm{VD}_{3}\right)$, and changed medium once every $3 \mathrm{~d}$. After $9 \mathrm{~d}$ of culture, $0.5 \mathrm{~mL}$ DMEM medium in the lower chambers was changed and cultured $0.5 \mathrm{~mL}$ of $1.0 \times 10^{5} \mathrm{cell} / \mathrm{mL}$ MC3T3-E1 with DMEM medium (without RANKL, M$\mathrm{CSF}$, or $\mathrm{VD}_{3}$ ) in the upper chambers under a humidified atmosphere of $5 \% \mathrm{CO}_{2}$ at $37^{\circ} \mathrm{C}$, and after $24 \mathrm{~h}$, the different concentrations of geraniin and $10^{-8} \mathrm{~mol} / \mathrm{L}$ alendronate were added into the lower chambers for $2 \mathrm{~d}$. The upper chamber and the medium in the lower chambers were removed and washed twice with a pre-warmed PBS solution, fixed with $4 \%$ paraformaldehyde, washed with $0.25 \mathrm{~mol} / \mathrm{L}$ $\mathrm{NH}_{4} \mathrm{OH}$ solution. The bone resorption was visualized by $1 \%$ toluidine blue staining. The resorptive areas and pits were quantified using an Image Pro Plus analysis system; the regions were also observed by scanning electronic microscopy (SEM). OLC-OB were cultured and divided into seven groups as follows $(n=4$ per group): OLC group; OLC-OB co-culture group without any added substances; geraniin groups with $10^{-8}, 10^{-9}, 10^{-10}$, and $10^{-11} \mathrm{~mol} / \mathrm{L}$ of geraniin in OLC-OB co-culture system; positive control group with $10^{-8} \mathrm{~mol} / \mathrm{L}$ of alendronate in OLC-OB co-culture system.

The assay of bone resorption was based on the ability of isolated osteoclasts, OLC, and OLC-OB to absorb devitalized cortical bone in vitro (Ducy, Schinke, 
Karsenty, 2000; Teitelbaum, 2000). After $72 \mathrm{~h}$ of culture with osteoclasts, and $4 \mathrm{~d}, 7 \mathrm{~d}, 12 \mathrm{~d}$, and $15 \mathrm{~d}$ of culture with OLC and OLC-OB, the bovine bone slices were removed and fixed with $4 \%$ paraformaldehyde solution for $10 \mathrm{~min}$. After the adherent cells were removed by treating the bovine bone slices with $0.25 \mathrm{~mol} / \mathrm{L} \mathrm{NH}_{4} \mathrm{OH}$ solution, the resorption areas and pits were visualized as described above.

\section{Immunocytochemical and in situ hybridization analysis}

Immunocytochemical and in situ hybridization analysis of OPG/RANKL was performed according to the kit's specifications. The values of integral optical density [IOD, equivalent to area of staining $\times$ mean optical density (MOD)] and MOD of positive cells in the visual field were detected by using a graphic analysis system. The slices were coated with $0.1 \%$ poly-L-Lysine, and one slice placed in each well of 24-well plate. The cells were cultured in the 24-well plate with poly-L-Lysine-coated slices for $24 \mathrm{~h}$. Briefly, the slices were washed thrice with PBS, fixed in 4\% paraformaldehyde solution for 20-30 min, then treated with the $0.5 \%$ Triton $\mathrm{X}-100$ for 20 min and $3 \% \mathrm{H}_{2} \mathrm{O}_{2}$ for 10 min to inactivate endogenous peroxidase, and closed with $5 \%$ sheep serum for $10 \mathrm{~min}$. The slices were incubated overnight with a 1:400 dilution of OPG rabbit anti mouse polyclonal antibody or RANKL goat anti mouse polyclonal antibody, and finally stained with DAB. Osteoblasts were cultured and divided into seven groups as above ( $n=4$ per group). PBS instead of the first antibody was used as the negative control. IOD and MOD of positively stained cells were examined as described above.

\section{Statistical analysis}

Data are expressed as mean \pm standard deviation (S.D.). Statistical comparisons were made by analysis of variance (ANOVA) and Student's $t$-test. Differences were considered statistically significant when $P$ was less than 0.05 .

\section{RESULTS}

\section{Effects of geraniin on proliferation and differentiation of osteoblasts}

Compared with the control group, there was no significant difference between geraniin $\left(10^{-7}\right.$ and $10^{-8}$ $\mathrm{mol} / \mathrm{L})$ groups $(P>0.05)$ (Figure 2$)$. However, the
OD values of osteoblasts in alendronate and geraniin $\left(10^{-9}\right.$ to $\left.10^{-12} \mathrm{~mol} / \mathrm{L}\right)$ groups were significantly higher $(P<0.01)$ than those in control group. Furthermore, $10^{-6} \mathrm{~mol} / \mathrm{L}$ geraniin markedly decreased the OD values $(P<0.05)$ compared with the control group. $10^{-11}$ and $10^{-12} \mathrm{~mol} / \mathrm{L}$ geraniin showed a similar potency compared with alendronate. As presented in Figure 3A, 3B, and 4, compared with the mechanical method ( $3 \mathrm{~d})$, the numbers of $\mathrm{mOC}$ and $\mathrm{pOC}$ were significantly higher than those in the induction method $(4 \mathrm{~d})(P<0.01)$, but lower than that in the induction method ( $7 \mathrm{~d}, 12 \mathrm{~d}$, and $15 \mathrm{~d}$, respectively) $(P<0.01)$. However, the numbers of $\mathrm{mOC}$ and $\mathrm{pOC}$ in the induction method (4d) were significantly lower $(P<0.01)$ than those in the induction method $(7 \mathrm{~d}, 12 \mathrm{~d}$, and $15 \mathrm{~d}$, respectively). The numbers of $\mathrm{mOC}$ and $\mathrm{pOC}$ in the induction method ( $7 \mathrm{~d})$ were significantly lower $(P<0.01)$ than those in the induction method (12 d and $15 \mathrm{~d})$. FI was different between the mechanical method group and the induction method groups. As shown in Figure 3C, induction method ( $4 \mathrm{~d}$ and $7 \mathrm{~d}$ ) significantly reduced FI as compared with the mechanical method $(3 \mathrm{~d})(P<0.05)$. Induction method (12 d and $15 \mathrm{~d})$ increased FI as compared with the mechanical method ( $3 \mathrm{~d})$ and induction method ( $4 \mathrm{~d}$ and $7 \mathrm{~d})$ $(P<0.01)$. As shown in Figure 4, the cellular morphology of osteoblasts had obvious difference between the mechanical and inductive methods.

\section{Effects of geraniin on bone resorption in OLC-OB co-culture system}

The bone resorptive areas were significantly different between the control groups (OLC and OLC-OB co-culture) $(P<0.01)$ (Figure 5A). In OLC and OLC-OB co-culture system, the bone resorptive areas in alendronate and geraniin $\left(10^{-8}\right.$ to $\left.10^{-10} \mathrm{~mol} / \mathrm{L}\right)$ groups were significantly decreased $(P<0.01)$ than those of the control groups. $10^{-8}$ $\mathrm{mol} / \mathrm{L}$ geraniin and alendronate showed similar abilities to decrease the resoptive areas.

There was no significant difference in the number of bone resorptive pits between the control (OLC and OLC-OB co-culture) and $10^{-11} \mathrm{~mol} / \mathrm{L}$ geraniin groups. Geraniin $\left(10^{-8}\right.$ and $\left.10^{-9} \mathrm{~mol} / \mathrm{L}\right)$ and alendronate significantly decreased the number of bone resorptive pits compared with control groups $(P<0.01) .10^{-8} \mathrm{~mol} / \mathrm{L}$ geraniin and alendronate also showed similar abilities to decrease the bone resorptive pits (Figure 5B).

\section{Protein and mRNA expression of OPG and RANKL}

There was no significant difference in the MOD values in OPG positive cells between the control and 


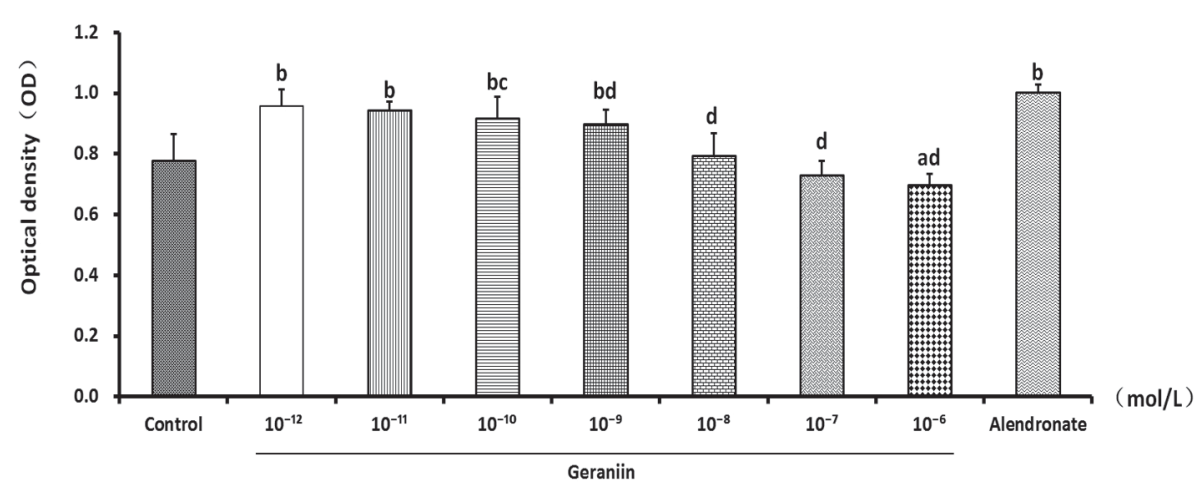

FIGURE 2 - Effects of geraniin on proliferation of osteoblasts. Values are mean \pm S.D. $(n=6)^{\mathrm{a}} P<0.05,{ }^{\mathrm{b}} P<0.01$ versus control group, ${ }^{\mathrm{c}} P<0.05,{ }^{\mathrm{d}} P<0.01$ versus alendronate group.
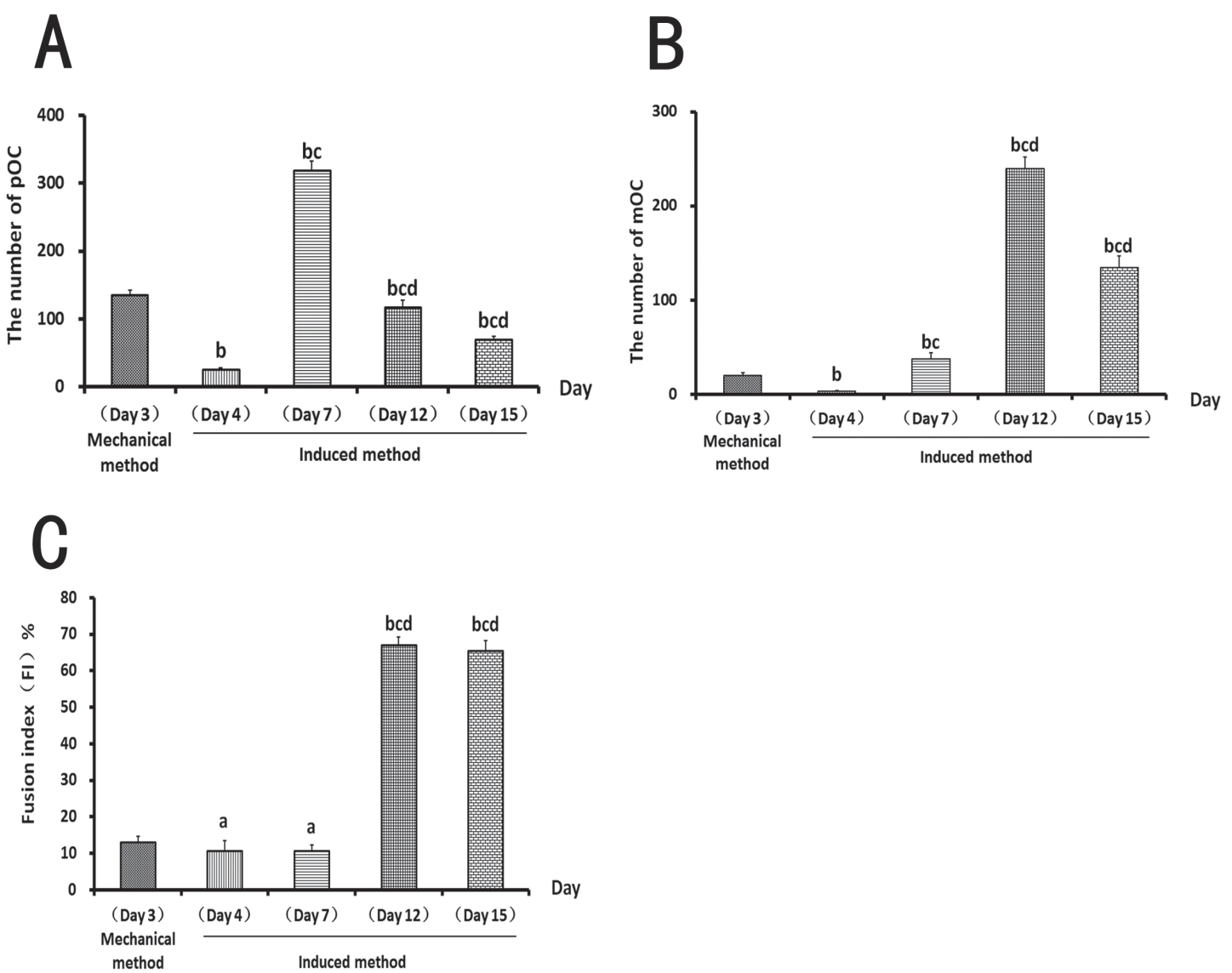

FIGURE 3 - Comparison of osteoclast differentiation between induction and mechanical procedure. (A) The numbers of pOC were significantly higher than those in the induction method (4 d), but lower than that in the induction method $(7 \mathrm{~d}, 12 \mathrm{~d}$, and $15 \mathrm{~d}$, respectively). The numbers of pOC in the induction method ( $4 \mathrm{~d})$ were significantly lower than those in the induction method ( $7 \mathrm{~d}, 12 \mathrm{~d}$, and $15 \mathrm{~d}$, respectively). The numbers of pOC in the induction method ( $7 \mathrm{~d}$ ) were significantly lower than those in the induction method (12 d and $15 \mathrm{~d}$ ). (B) The numbers of mOC were significantly higher than those in the induction method (4d), but lower than that in the induction method ( $7 \mathrm{~d}, 12 \mathrm{~d}$, and $15 \mathrm{~d}$, respectively). However, the numbers of mOC in the induction method (4 d) were significantly lower than those in the induction method ( $7 \mathrm{~d}, 12 \mathrm{~d}$, and $15 \mathrm{~d}$, respectively). The numbers of mOC in the induction method ( $7 \mathrm{~d}$ ) were significantly lower than those in the induction method ( $12 \mathrm{~d}$ and $15 \mathrm{~d})$. (C) Induction method ( $4 \mathrm{~d}$ and $7 \mathrm{~d})$ groups reduced FI as compared with the mechanical method ( $3 \mathrm{~d})$ group. Induction method (12 d and $15 \mathrm{~d})$ groups increased FI as compared with the mechanical method ( $3 \mathrm{~d})$ group and induction method ( $4 \mathrm{~d}$ and $7 \mathrm{~d}$ ) groups. Values are mean $\pm \operatorname{S} . \mathrm{D}(n=4)$. ${ }^{\mathrm{a}} P<0.05,{ }^{\mathrm{b}} P<0.01$ versus mechanical for $3 \mathrm{~d}$ group, ${ }^{\mathrm{c}} P<0.05$ versus induction for $4 \mathrm{~d}$ group, ${ }^{\mathrm{d}} P<0.01$ versus induction for $7 \mathrm{~d}$ group. 


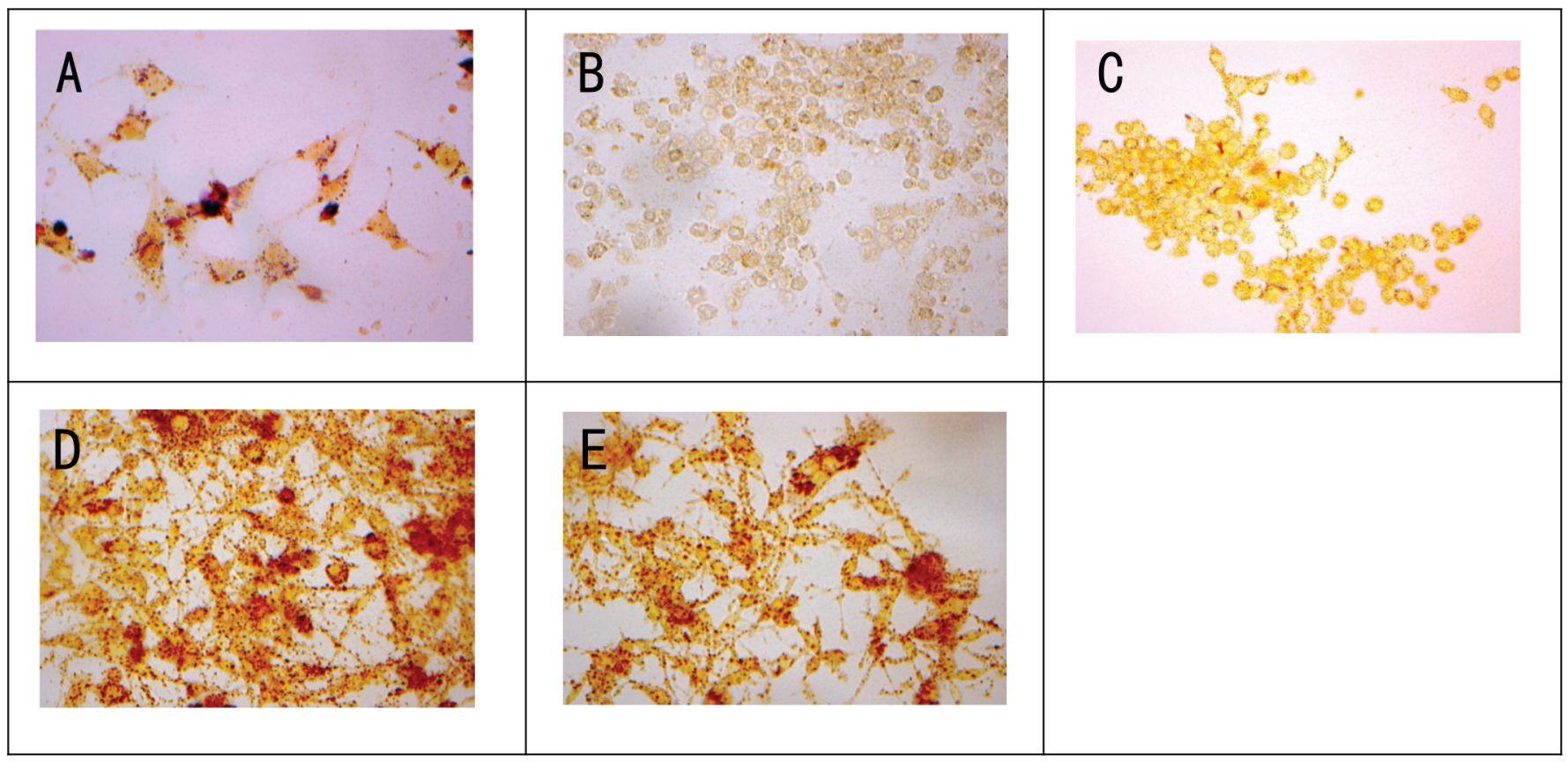

FIGURE 4 - Comparison of mOC between mechanical and inductive procedure by TRAP staining $(\times 400)$. (A) Mechanical method for $3 \mathrm{~d}$. (B) Inductive method for $4 \mathrm{~d}$. (C) Inductive method $7 \mathrm{~d}$. (D) Inductive method for $12 \mathrm{~d}$. (E) Inductive method for $15 \mathrm{~d}$.
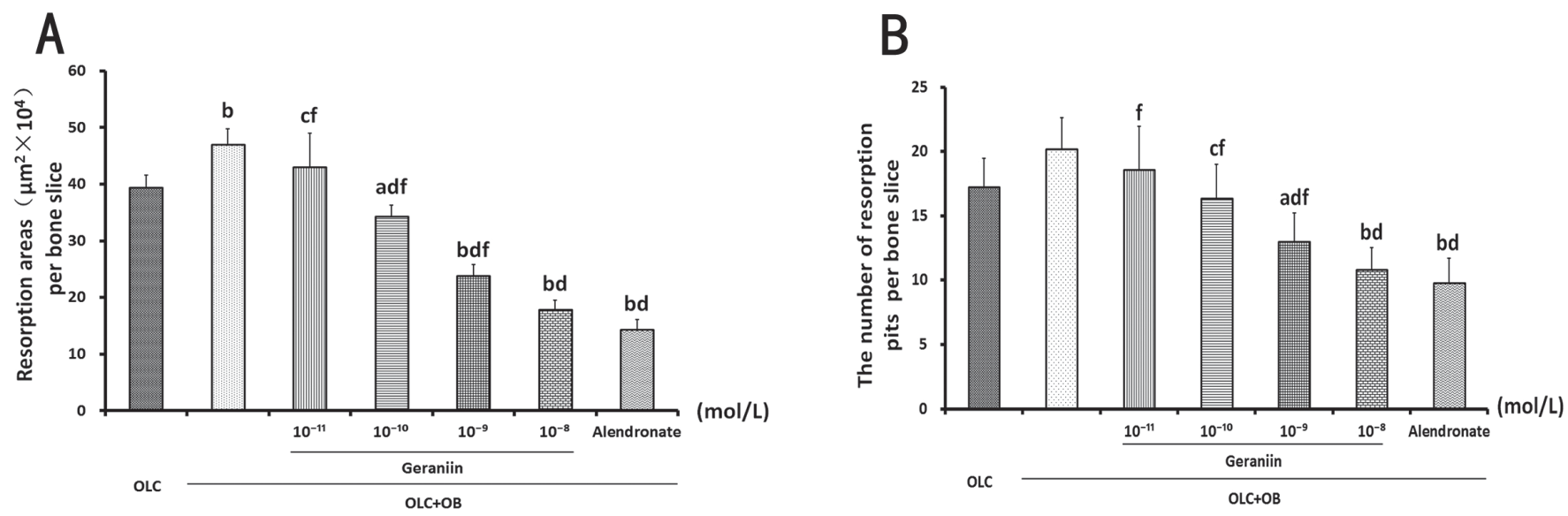

FIGURE 5 - Effects of geraniin on bone resorption in OLC-OB co-culture system. Geraniin $\left(10^{-11}-10^{-8} \mathrm{~mol} / \mathrm{L}\right)$ concentrationdependently inhibited the resorption areas (A) and the number of resorption pits (B) per bone slice. Values are mean \pm S.D. ${ }^{a} P<0.05$, ${ }^{\mathrm{b}} P<0.01$ versus OLC group, ${ }^{\mathrm{c}} P<0.05,{ }^{\mathrm{d}} P<0.01$ versus $\mathrm{OLC}+\mathrm{OB}$ group, ${ }^{\mathrm{e}} P<0.05,{ }^{\mathrm{f}} P<0.01$ versus between medicated group and alendronate group.

geraniin $\left(10^{-11} \mathrm{~mol} / \mathrm{L}\right)$ groups (Figure $6 \mathrm{~A}$ and Figure $\left.8 \mathrm{~A}\right)$. In comparison, geraniin $\left(10^{-11}\right.$ to $\left.10^{-8} \mathrm{~mol} / \mathrm{L}\right)$ did increase IOD and MOD values of positive cells for the protein expression of OPG in a concentration-dependent manner (Figure 6A, B, and Figure 8A). 10-8 $\mathrm{mol} / \mathrm{L}$ geraniin showed similar capacity for up-regulating the protein expression of OPG compared with alendronate. As presented in Figure 6C, D, and Figure 8B, no significant differences were shown in IOD and MOD values of positive cells for OPG mRNA expression between the control and geraniin groups $\left(10^{-10}\right.$ and $\left.10^{-11} \mathrm{~mol} / \mathrm{L}\right)$. Compared with the control and geraniin groups $\left(10^{-10}\right.$ and $\left.10^{-11} \mathrm{~mol} / \mathrm{L}\right)$, $10^{-8}$ and $10^{-9} \mathrm{~mol} / \mathrm{L}$ geraniin and alendronate, however, significantly increased IOD and MOD values of positive cells for mRNA expression of OPG.

In contrast, there was no significant difference in MOD values of protein expression in RANKL positive cells between the control and geraniin $\left(10^{-10}\right.$ and $10^{-11}$ $\mathrm{mol} / \mathrm{L}$ ) groups and IOD between the control and geraniin groups $\left(10^{-11} \mathrm{~mol} / \mathrm{L}\right)$ ( Figure $7 \mathrm{~A}, \mathrm{~B}$, and Figure $\left.9 \mathrm{~A}\right)$. In comparison, geraniin $\left(10^{-9}\right.$ and $\left.10^{-8} \mathrm{~mol} / \mathrm{L}\right)$ decreased IOD and MOD values of positive cells for RANKL protein 
expression in a concentration-dependent manner. $10^{-8}$ $\mathrm{mol} / \mathrm{L}$ geraniin also showed similar capacity for downregulation of RANKL protein expression compared with alendronate. As shown in Figure 7C, D and Figure 9B, there were no significant differences in IOD and MOD values of mRNA expression in RANKL positive cells between the control and geraniin groups $\left(10^{-10}\right.$ and $10^{-11}$ $\mathrm{mol} / \mathrm{L})$. Compared with the control and geraniin groups $\left(10^{-10}\right.$ and $\left.10^{-11} \mathrm{~mol} / \mathrm{L}\right), 10^{-8}$ and $10^{-9} \mathrm{~mol} / \mathrm{L}$ geraniin and alendronate, however, significantly increased IOD and MOD values of positive cells for mRNA expression of RANKL. $10^{-8} \mathrm{~mol} / \mathrm{L}$ geraniin also showed a similar ability compared with alendronate.

\section{DISCUSSION}

Bone remodelling is a continuous physiological process that occurs in adult skeleton in which bone resorption is followed by new bone formation. Bone cells that are responsible for this coupled process include bone-resorbing cells (osteoclasts, which are derived from haematopoietic cells of the monocyte/macrophage lineage) and bone-forming cells (osteoblasts, which are of mesenchymal origin).

RANKL/RANK/OPG system is one of the most important signal transduction pathways in the process of osteoclastic differentiation in recent years (Jules, Ashley, Feng, 2010). Osteoclasts play a key role in bone remodeling, and bone resorption through the binding of RANKL to its receptor-RANK, on the surface of pre-osteoclast cells. On the other hand, osteoblasts secrete a soluble decoy receptor (osteoprotegerin) that blocks RANK/RANKL interaction by binding to RANKL and thus, prevents the differentiation and activation of osteoclasts (Kiechl et al., 2006; Trouvin, Goeb, 2010). The ratio of these products, therefore, may modulate the ability of osteoblasts to stimulate the differentiation and activation of osteoclasts, as well as the degree of bone resorption (Eghbali-Fatourechi et al., 2003). It is of great interest that new and effective drug reducing the number and activity of osteoclasts, simultaneously increasing the number and activity of osteoblasts, may be designed to treat osteoporosis.

In our preliminary studies, we found that geraniin has an inhibitory effect on bone resorption only by
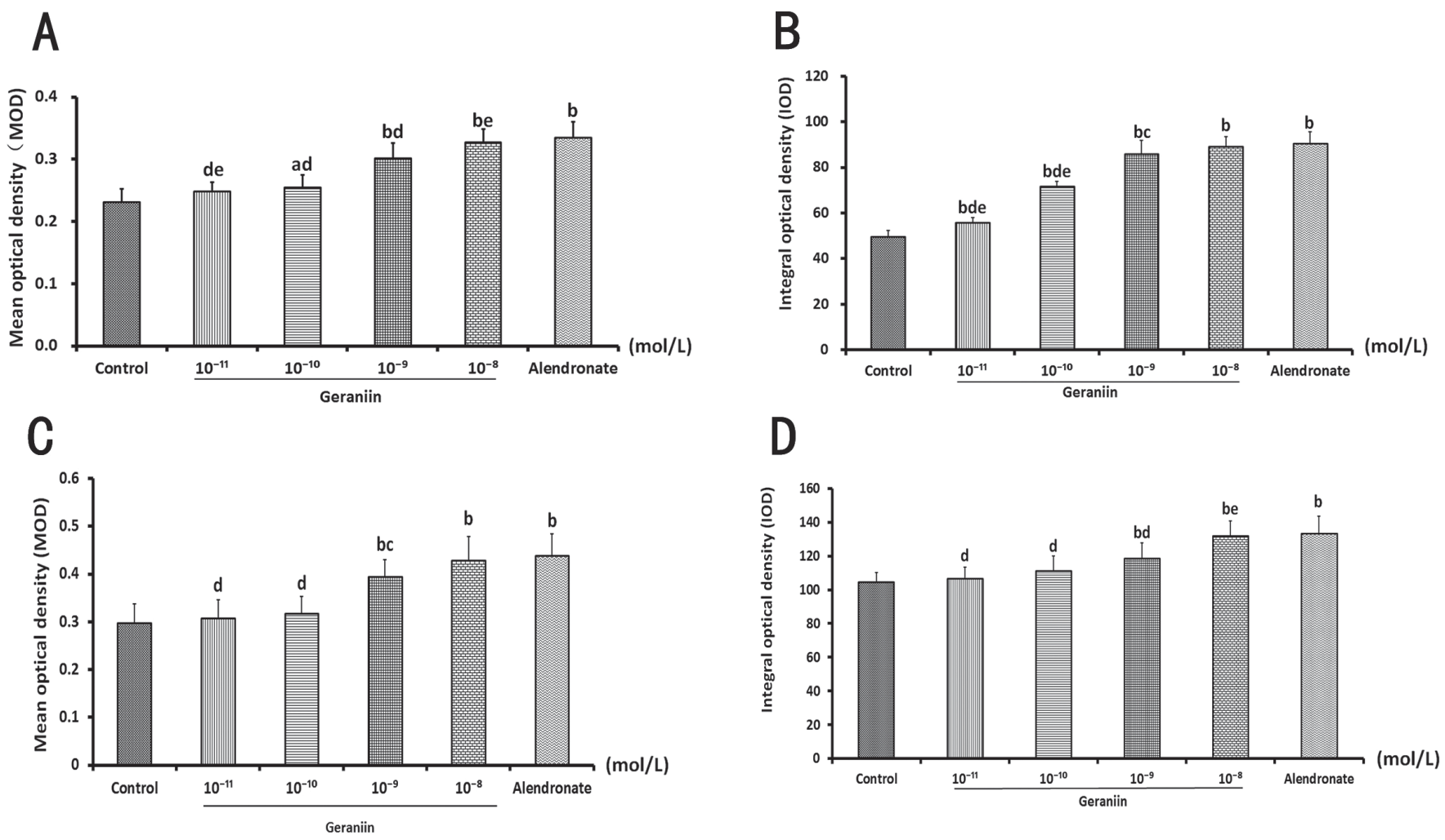

FIGURE 6 - Effect of geraniin on protein and mRNA expression of OPG in osteoblasts. Geraniin $\left(10^{-11}\right.$ to $\left.10^{-8} \mathrm{~mol} / \mathrm{L}\right)$ increased MOD (A) and IOD (B) values of OPG protein expression in positive cells. Geraniin $\left(10^{-11}\right.$ to $10^{-8}$ mol/L) concentration-dependently increased MOD (C) and IOD (D) values of OPG mRNA expression in positive cells. Values are mean $\pm \mathrm{S} . \mathrm{D} .{ }^{a} P<0.05,{ }^{\mathrm{b}} P<0.01$ versus control group, ${ }^{c} P<0.05$, ${ }^{\mathrm{d}} P<0.01$ versus alendronate group, ${ }^{e} P<0.05$ versus between geraniin $\left(10^{-11}\right.$ to $\left.10^{-8} \mathrm{~mol} / \mathrm{L}\right)$ groups . One-way ANOVA followed by Student's $t$-test. 

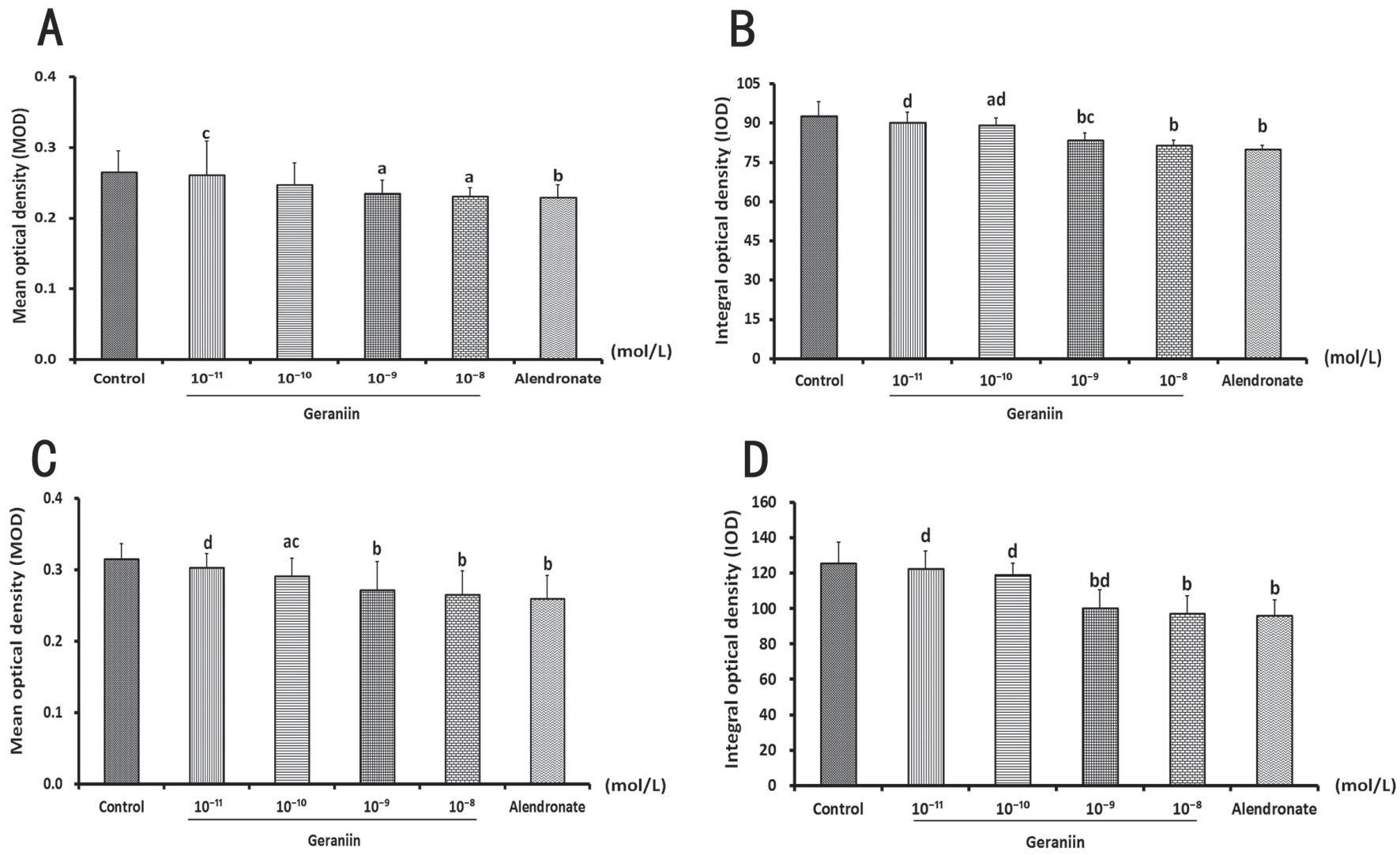

FIGURE 7 - Effect of geraniin on protein and mRNA expression of RANKL in osteoblasts. Geraniin $\left(10^{-11}\right.$ to $10^{-8}$ mol/L) concentration-dependently decreased IOD (A) and MOD (B) values of RANKL protein expression in positive cells. Geraniin (10 ${ }^{-11}$ to $10^{-8} \mathrm{~mol} / \mathrm{L}$ ) concentration-dependently decreased MOD $(\mathrm{C})$ and IOD (D) values of RANKL mRNA expression in positive cells. ${ }^{a} P<0.05,{ }^{\mathrm{b}} P<0.01$ versus control group, ${ }^{c} P<0.05,{ }^{\mathrm{d}} P<0.01$ versus alendronate group, ${ }^{e} P<0.05$ versus between geraniin $\left(10^{-11}\right.$ to $\left.10^{-8} \mathrm{~mol} / \mathrm{L}\right)$ groups. One-way ANOVA followed by Student's $t$-test.

osteoclasts in vitro (He et al., 2013). And in this study, we also found that geraniin has an inhibitory effect on bone resorption through regulating the proliferation and differentiation of the osteoblasts in vitro.

The morphology of MC3T3-E1, through primary culture, is similar to osteoblasts. The higher activity of alkaline phosphatase (ALP) and the ability of mineralization reflect the two typical characteristics of differentiation and maturation of osteoblasts in vitro. In this study, the positive rate of ALP staining of MC3T3-E1 cells was more than $94 \%$, and with the times of cell passages increasing, the positive rate of ALP was not decreased, indicating that multiple passage of the cells shows not significantly influence on the ability to secret alkaline phosphatase, and also suggesting that the obtained cells were mature osteoblasts. Thus, MC3T3-E1 can replace the primary cultured osteoblast as the cells for our further study. $10^{-12}-10^{-9} \mathrm{~mol} / \mathrm{L}$ geraniin significantly stimulated the proliferation of osteoblasts in a concentration dependent manner, while $10^{-6} \mathrm{~mol} / \mathrm{L}$ geraniin inhibited the proliferation of osteoblasts. It is suggested that the effects of geraniin on proliferation of osteoblasts show bi-directional nature.

RAW264.7 cells are the only osteoclast precursor cells and share an even deeper homology with MC3T3-E1 cells (Sanchez-Fernandez et al., 2008). It is reported that RAW264.7 cells can express the marker gene of osteoclastic phenotype and shows the function of bone resorption (Walsh et al., 2003). In this study, OLCs were successfully induced from RAW264.7 by RANKL and M-CFS. The fusion index (FI), presented as the average number of mOC cultured in vitro, indicates the number of cells that participate in cell fusion and mOC formation. TRAP staining, morphological observation and formation of bone resorption lacunae have been used as the important and reliable method for identifying OC (Walsh et al., 2003). Our results revealed that geraniin significantly decreased the number of $\mathrm{mOC}$ and $\mathrm{pOC}$, and the inhibited osteoclast formation due to its suppressing the fusion of pOC into mOC.

In our study, $\mathrm{OC}, \mathrm{OB}$, and bovine bone slices were cultured together to observe the effect of geraniin on 


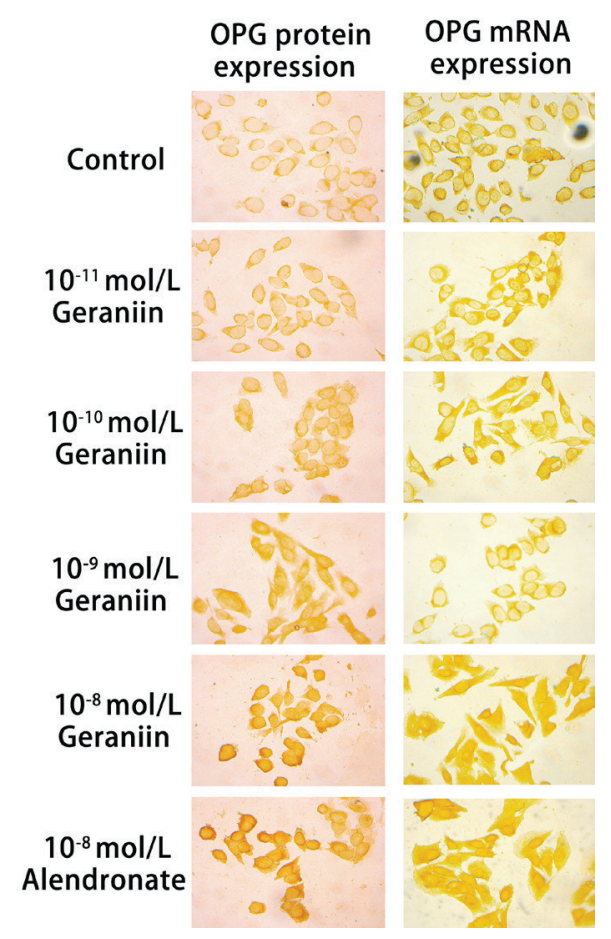

FIGURE 8 - Representative images of immunocytochemistry staining for OPG protein expression (A) and in situ hybridization staining for OPG mRNA expression(B). Geraniin $\left(10^{-11}-10^{-8} \mathrm{~mol} / \mathrm{L}\right)$ increased IOD and MOD values of OPG mRNA and protein expression in positive cells.

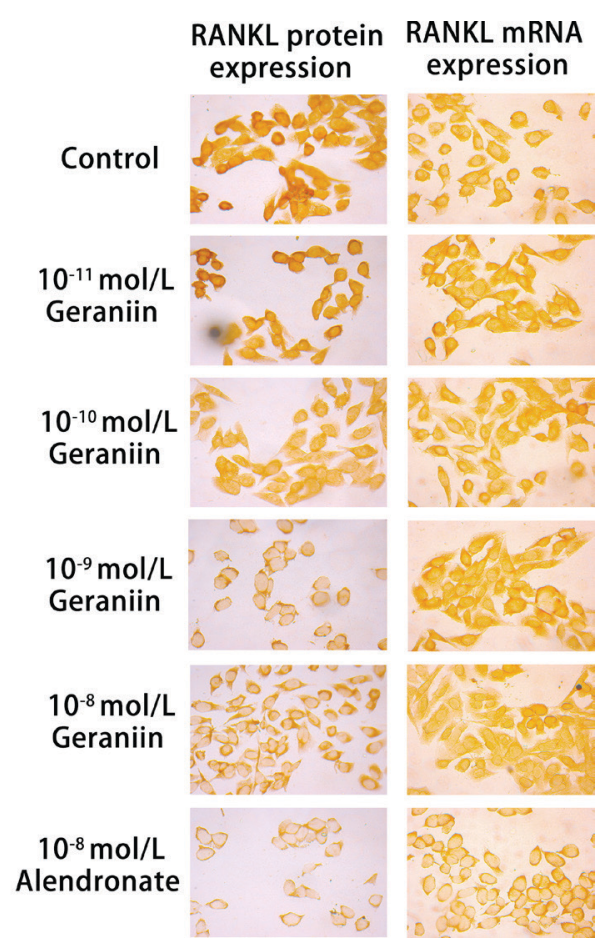

FIGURE 9 - Representative images of immunocytochemistry staining for RANKL protein expression (A) and in situ hybridization staining for RANKL mRNA expression(B). Geraniin $\left(10^{-11}-10^{-8} \mathrm{~mol} / \mathrm{L}\right)$ decreased IOD and MOD values of RANKL mRNA and protein expression in positive cells. osteoclastic bone resorption in OC-OB co-culture system. The results showed that geraniin $\left(10^{-10}\right.$ to $\left.10^{-8} \mathrm{~mol} / \mathrm{L}\right)$ could decrease the resorptive area and the number of resorptive pits in a concentration-dependent manner, suggesting that geraniin has an inhibitory effect on bone resorption in this OC-OB co-cultured system.

As one of major signaling pathways, RANKL/ RANK/OPG is responsible for the bone resorption and osteogenesis through the interaction between osteoblasts and osteoclasts. The present study showed that geraniin decreases IOD and MOD of RANKL positive cells, while increased IOD and MOD of OPG, suggesting that geraniin can regulate the mRNA and protein expression of $\mathrm{OPG} /$ RANKL.

\section{CONCLUSION}

Geraniin shows a bi-directional nature on the proliferation of MC3T3-E1, inhibits the proliferation and differentiation of osteoclasts, and suppresses osteoclastic bone absorption. Upregulating OPG expression and downregulating RANKL expression may be responsible for its modulating the signaling pathway of OPG/RANKL.

\section{ACKNOWLEDGEMENTS}

This work was supported by the National Natural Science Foundation of China (grant No.:81660613, 81260493), and the Natural Science Foundation of Yunnan Province, P.R. China (grant No.: 2015FA021).

\section{REFERENCES}

Czekanska EM, Stoddart MJ, Ralphs JR, Richards RG, Hayes JS. A phenotypic comparison of osteoblast cell lines versus human primary osteoblasts for biomaterials testing. J Biomed Mater Res A. 2014;102(8):2636-2643.

Denlinger LC, Fisette PL, Garis KA, Kwon G, Vazquez-Torres A, Simon AD, et al. Regulation of inducible nitric oxide synthase expression by macrophage purinoreceptors and calcium. J Biol Chem. 1996;271(1):337-342.

Ducy P, Schinke T, Karsenty G. The osteoblast: a sophisticated fibroblast under central surveillance. Science. 2000;289(5484):1501-1504.

Eghbali-Fatourechi G, Khosla S, Sanyal A, Boyle WJ, Lacey DL, Riggs BL. Role of RANK ligand in mediating increased bone resorption in early postmenopausal women. J Clin Invest. 2003;111(8):1221-1230. 
He B, Hu M, Li SD, Yang XT, Lu YQ, Liu JX, et al. Effects of geraniin on osteoclastic bone resorption and matrix metalloproteinase-9 expression. Bioorg Med Chem Lett. 2013;23(3):630-634.

Ito H. Metabolites of the ellagitannin geraniin and their antioxidant activities. Planta Med. 2011;77(11):1110-1115.

Janckila AJ, Yam LT. Biology and clinical significance of tartrate-resistant acid phosphatases: new perspectives on an old enzyme. Calcif Tissue Int. 2009;85(6):465-483.

Jules JJ, Ashley W, Feng X. Selective targeting of RANK signaling pathways as new therapeutic strategies for osteoporosis. Expert Opin Ther Targets. 2010;14(9):923-934.

Kiechl S, Werner P, Knoflach M, Furtner M, Willeit J, Schett G. The osteoprotegerin/RANK/RANKL system: a bone key to vascular disease. Expert Rev Cardiovasc Ther. 2006;4(6):801811.

Kim JL, Li HM, Kim YH, Lee YJ, Shim JH, Lim SS, et al. Osteogenic activity of yellow flag iris (Iris pseudacorus) extract modulating differentiation of osteoblasts and osteoclasts. Am J Chin Med. 2012;40(6):1289-1305.

Kupeli E, Tatli II, Akdemir ZS, Yesilada E. Estimation of antinociceptive and anti-inflammatory activity on Geranium pratense subsp. finitimum and its phenolic compounds. J Ethnopharmacol. 2007;114(2):234-240.

Lee YS, Choi EM. Apocynin stimulates osteoblast differentiation and inhibits bone-resorbing mediators in MC3T3-E1 cells. Cell Immunol. 2011;270(2):224-229.

Luan J, Cui Y, Zhang Y, Zhou X, Zhang G, Han J. Effect of CXCR4 inhibitor AMD3100 on alkaline phosphatase activity and mineralization in osteoblastic MC3T3-E1 cells. Biosci Trends. 2012;6(2):63-69.
Ryoo HM, van Wijnen AJ, Stein JL, Lian JB, Stein GS. Detection of a proliferation specific gene during development of the osteoblast phenotype by mRNA differential display. J Cell Biochem. 1997;64(1):106-116.

Sanchez-Fernandez MA, Gallois A, Riedl T, Jurdic P, Hoflack B. Osteoclasts control osteoblast chemotaxis via PDGF-BB/ PDGF receptor beta signaling. PLoS One. 2008;3(10):e3537.

Simonet WS, Lacey DL, Dunstan CR, Kelley M, Chang MS, Luthy R, et al. Osteoprotegerin: a novel secreted protein involved in the regulation of bone density. Cell. 1997;89(2):309319.

Teitelbaum SL. Bone resorption by osteoclasts. Science. 2000;289(5484):1504-1508.

Trouvin AP, Goeb V. Receptor activator of nuclear factorkappaB ligand and osteoprotegerin: maintaining the balance to prevent bone loss. Clin Interv Aging. 2010;5:345-354.

Walsh NC, Cahill M, Carninci P, Kawai J, Okazaki Y, Hayashizaki Y, et al. Multiple tissue-specific promoters control expression of the murine tartrate-resistant acid phosphatase gene. Gene. 2003;307:111-123.

Yang Y, Zhang L, Fan X, Qin C, Liu J. Antiviral effect of geraniin on human enterovirus 71 invitro and in vivo. Bioorg Med Chem Lett. 2012;22(6):2209-2211.

Received for publication on $20^{\text {th }}$ September 2017 Accepted for publication on $08^{\text {th }}$ January 2018 